\title{
Corela
}

Cognition, représentation, langage

$1-2 \mid 2003$

Vol. $1, \mathrm{n}^{\circ} 2$

\section{La contingence des faits linguistiques : réflexions sur la variation et le changement}

\section{Pierre Larrivée}

\section{OpenEdition}

\section{Journals}

Édition électronique

URL : http://journals.openedition.org/corela/688

DOI : $10.4000 /$ corela.688

ISSN : $1638-573 \mathrm{X}$

Éditeur

Cercle linguistique du Centre et de l'Ouest - CerLICO

Référence électronique

Pierre Larrivée, "La contingence des faits linguistiques : réflexions sur la variation et le changement », Corela [En ligne], 1-2 | 2003, mis en ligne le 01 décembre 2003, consulté le 22 avril 2019. URL : http:// journals.openedition.org/corela/688; DOI : 10.4000/corela.688

Ce document a été généré automatiquement le 22 avril 2019

\section{(c) (i) (2)(2)}

Corela - cognition, représentation, langage est mis à disposition selon les termes de la licence Creative Commons Attribution - Pas d'Utilisation Commerciale - Partage dans les Mêmes Conditions 4.0 International. 


\title{
La contingence des faits linguistiques: réflexions sur la variation et le changement
}

\author{
Pierre Larrivée
}

\section{Introduction ${ }^{1}$}

1 Comme toute science, celle du langage a pour objectif l'identification de déterminismes généraux expliquant la variété des manifestations observables des phénomènes. Cette recherche de déterminismes trouve une illustration dans la plupart des modèles d'analyse de la linguistique moderne, depuis Saussure jusqu'à Chomsky. Ces modèles se sont cependant essentiellement préoccupés de déterminismes catégoriques et généraux, tant et si bien que les manifestations de la variation et du changement ne semblent guère $\mathrm{y}$ trouver place. C'est la situation respective des contingences et des nécessités que cherchent à articuler les présentes réflexions.

La dichotomie entre nécessités et contingences permet surtout de renouveler la façon de penser les lieux de transition entre la langue et la parole, la compétence et la performance. Ce renouvellement me semble crucial à un moment où la maîtrise méthodologique qu'a acquise la linguistique lui permet de soulever maints problèmes qui ne semblent pas trouver leur place dans les modèles classiques. Il me semble approprié à une époque où maintes disciplines s'interrogent sur la notion de phénomène contingent. Afin de soutenir la réflexion et sans le moins du monde prétendre à une connaissance spécialiste, je me réfèrerai ainsi à des observations apparemment établies et particulièrement parlantes des sciences de la vie et de la culture. De même, les observations sur le langage serviront à l'appréhension des points de contact entre possibilités et actualisations linguistiques. C'est donc dans le débat sur la nature des représentations linguistiques, sur les notions de langue et de système, que doit être située la présente contribution. 


\section{La régularité des systèmes: l'exemple des indéfinis}

L'analyse saussurienne pose que les rapports d'opposition entre les unités constitutives d'un idiome en définissent le contenu. Le contenu des unités n'a pas d'existence propre hors de ces rapports différentiels caractérisant un idiome. Poussée à ses conséquences ultimes $^{2}$, cette perspective suppose qu'un idiome est constitué de paradigmes stables et optimaux. Si le tout régit les parties, un même axe d'opposition devrait affecter également toutes les formes d'un paradigme, chaque forme devrait s'opposer à toutes les autres et toutes les oppositions devraient être réalisées selon des modes proportionnels. Or, ces prédictions sont invalidées par le phénomène des paradigmes imparfaits et par les emplois créatifs des formes.

Les paradigmes imparfaits peuvent être illustrés par les pronoms dits indéfinis :

\begin{tabular}{|l|l|l|l|l|l|}
\hline Humains & quelqu'un & lui & autrui & la même personne & qui \\
\hline Inanimés & quelque chose & ça & autre chose & la même chose & quoi \\
\hline Locatifs & quelque part & ici & ailleurs & au même endroit & où \\
\hline Temporels & un jour & main-tenant & un autre jour & le même jour & quand \\
\hline
\end{tabular}

5 (suite)

\begin{tabular}{|l|l|l|l|l|l|l|}
\hline Humains & chacun & $\begin{array}{l}\text { toute } \\
\text { personne }\end{array}$ & n'importe qui & $\begin{array}{l}\text { qui que ce } \\
\text { soit }\end{array}$ & $\begin{array}{l}\text { pas grand- } \\
\text { monde }\end{array}$ & personne \\
\hline Inanimés & $\begin{array}{l}\text { chaque } \\
\text { chose }\end{array}$ & toute chose & $\begin{array}{l}\text { n'importe } \\
\text { quoi }\end{array}$ & $\begin{array}{l}\text { quoi que ce } \\
\text { soit }\end{array}$ & $\begin{array}{l}\text { pas grand- } \\
\text { chose }\end{array}$ & Rien \\
\hline Locatifs & $\begin{array}{l}\text { chaque } \\
\text { endroit }\end{array}$ & partout & n'importe où & où que ce soit & $\begin{array}{l}\text { ? pas grand- } \\
\text { place }\end{array}$ & $\begin{array}{l}\text { nulle } \\
\text { part }\end{array}$ \\
\hline Temporels & chaque fois & toujours & $\begin{array}{l}\text { n'importe } \\
\text { quand }\end{array}$ & $\begin{array}{l}\text { ? quand que } \\
\text { ce soit }\end{array}$ & pas souvent & jamais. \\
\hline
\end{tabular}

Table 1

6 Au niveau des formes, on observe des sémiotisations non proportionnelles : la formation morphologiquement simple de autrui et de ailleurs se distingue de celle complexe de autre chose et de celle syntagmatique de un autre jour. Si quelquefois est de formation proportionnelle à quelqu'un, sa distribution semble plus particulière que celle de un jour. Au niveau de l'ensemble, les unités ne s'excluent pas toujours mutuellement: la case de personne pourrait être occupée par nul et aucun; de même, des formes autre part (pour ailleurs) (Damourette et Pichon 1940, t.7 : 147), pas grand part et aucune part (pour nulle part ) (Damourette et Pichon 1940, t.7: 146 et 139), aucune fois (pour jamais) (Damourette et 
Pichon 1940, t.7: 138-139), et en français médiéval, outre nullui (pour personne) (Damourette et Pichon 1940, t.7 : 147), et la synonymie de néant et rien, on trouve pour le locatif par exemple en nulle part, de nule part, en nule place, en nul leu et pour le temporel nule fois et nul jour (Blanche-Benveniste 2002 : 2). De plus, les axes d'opposition ne sont pas tous réalisés, comme le montrent les domaines particulièrement défectifs de la cause et de la manière (Blanche-Benveniste 2002) ; les cases vides ne pourront être remplies que par des formations peu idiomatiques :

\begin{tabular}{|l|l|l|l|l|l|}
\hline Cause & quelque raison & $\begin{array}{l}\text { cette raison-là ; pour } \\
\text { ça la même }\end{array}$ & $\begin{array}{l}\text { une autre } \\
\text { raison }\end{array}$ & pourquoi \\
\hline Manière & $\begin{array}{l}\text { quelque } \\
\text { manière }\end{array}$ & $\begin{array}{l}\text { cette manière-là ; } \\
\text { ainsi }\end{array}$ & $\begin{array}{l}\text { une autre } \\
\text { façon }\end{array}$ & la même façon & comment \\
\hline
\end{tabular}

7 (suite)

\begin{tabular}{|l|l|l|l|l|l|l|}
\hline Cause & $\begin{array}{l}\text { chaque } \\
\text { raison }\end{array}$ & $\begin{array}{l}\text { toute } \\
\text { raison }\end{array}$ & $\begin{array}{l}\text { n'importe } \\
\text { quelle raison }\end{array}$ & $\begin{array}{l}\text { quelque raison } \\
\text { que ce soit }\end{array}$ & $\begin{array}{l}\text { pas grand } \\
\text { raison }\end{array}$ & $\begin{array}{l}\text { aucune } \\
\text { raison }\end{array}$ \\
\hline Manière & $\begin{array}{l}\text { chaque } \\
\text { manière }\end{array}$ & $\begin{array}{l}\text { toute } \\
\text { manière }\end{array}$ & $\begin{array}{l}\text { n'importe } \\
\text { comment }\end{array}$ & $\begin{array}{l}\text { de quelque } \\
\text { manière que ce } \\
\text { soit }\end{array}$ & $\begin{array}{l}\text { ?? pas grand } \\
\text { manière }\end{array}$ & $\begin{array}{l}\text { aucune } \\
\text { manière }\end{array}$ \\
\hline
\end{tabular}

Table 2

8 Au niveau sémantique, des formes conventionnelles sous les mêmes cases de différents domaines sont loin de se correspondre parfaitement: toute manière a une valeur pragmatique que n'a pas toute chose, comme n'importe quoi a des emplois métalinguistiques inconnus de n'importe qui. Les domaines eux-mêmes ne s'excluent pas mutuellement de façon absolue, comme le montre l'incertitude de la désignation des animaux entre humains et inanimés; une catégorisation d'inanimé peut s'appliquer aux humains dans certaines circonstances :

(1)a Plus stupide que Francesco, rien. (J. Folch-Ribas. 1991. Première noctune. Paris

: Laffont. 144)

(1b) Que t'as tué Simmer, parbleu ! [...]

Non! j'ai rien tué !...

(L.-F. Céline 1987. Nord. Paris : Gallimard. 579)

9 L'absence d'étanchéité entre les catégories ontologiques et les formes, les synonymies, les concurrences, les cases vides ou remplies par des circonlocutions, les formations non proportionnelles même dans le cas des unités hautement grammaticalisées que sont les indéfinis indiquent que les paradigmes linguistiques ne correspondent probablement pas à l'idéal du système.

10 Cette conclusion est de plus étayée par l'existence de la création de formes non conventionnelles, tant en français contemporain que dans d'autres synchronies (comme en atteste l'hapax en (3)) : 
(2)a.[...] la Désirade ont leurs habitués qui ne voudraient aller nulle autre part au monde. (apud Muller 1991 : 289)

(2)b En peu de temps, les fantômes du passé se manifestent de façons terrifiantes ce qui fait comprendre à ces quatre personnes pourquoi nul âme qui vive ne reste dans ce manoir, surtout la nuit dans le noir. Habité d'esprits maléfiques, le manoir de Hill leur dévoilera ses plus effroyables secrets.

(http://www.films.qc.ca/hauntinghillhouse.html)

(2)c Comme annoncé sur les cartes le secteur était vide de tout élément. Par conscience professionnelle Wagy quadrilla le secteur. Il découvrit un petit champ d'astéroïdes ferreux. Seulement quatre possédaient un emplacement planétaire et trois en possédaient deux. Tous ces emplacements étaient des emplacements miniers. Aucune âme qui vive ne fut repérée dans ce champ d'astéroïdes. Par logique de cartographie le champ fut nommé Alpha 4.16.

(http://www.chez.com/leymurien/Wagy/Wagy01.htm)

(2)d Il croyait n'avoir rien autre chose à faire. (Stendhal. 1964. Le Rouge et le Noir. Paris : Garnier-Flammarion. 194)

(3) Et piz y a des aucuns qui soubz ombrede leurs dis offices ou service font plusieurs extorcions et roberies, à maintes povres gens si comme les preneurs pour les seigneurs et telz gens. (Hapax relevé chez C. de Pizan, Le Livre de la paix, 1412, 84. Catherine Schnedecker et Sophie Prévost. 2002. «Aucun(e)(s)/d'aucun(e)(s)/les aucun(e)s: évolution du français médiéval au français moderne ". Séminaire Diachronie du français, IUF, 23.05.02. Document d'accompagnement, 18 pages, $p$. 3)

11 Ces formes ne devraient pas être produites s'il existait un système optimal d'oppositions épuisant le champ des possibles pour un état de langue. Leur production devrait être soit foncièrement incompréhensible, puisqu'elles n'appartiennent pas au système d'opposition censé définir les unités de l'idiome ; si elles y appartiennent, leur production dénonce un système intégralement distinct, les oppositions définitoires des unités étant modifiées de fond en comble par l'intervention d'une nouvelle unité forçant la restructuration des paradigmes (de Mauro 1969).

La considération des manifestations des signes appartenant aux ensembles les plus stables semble donc remettre en débat l'idéal d'un système immanent d'oppositions.

\section{La perfection du système computationnel : le paramètre du sujet nul}

13 Le caractère régulier des systèmes pourrait être préservé s'il est supposé que demeurent déterminants à un niveau sous-jacent les réseaux d'oppositions. Ces propriétés différentielles s'attacheraient à des réalisations de surface variables, conventionnelles ou non, sans expression nécessairement exclusive ou proportionnelle. Selon le programme minimaliste de Chomsky, le caractère capricieux des réalisations apparentes ne changerait rien à la perfection des représentations à un niveau abstrait (ce qui n'est pas sans rappeler le principe de suffisance expressive de Guillaume). Cette perfection s'exprime par la généralité des opérations du système computationnel de la syntaxe, et par le petit nombre de traits que vérifie ce système. La vérification des traits motive à elle seule l'entier de la syntaxe et le statut de trait fort ou faible rendrait compte de tous les changements, de toutes les variations linguistiques observables. L'explication de l'entier du changement et de la variation par un petit nombre de propriétés se trouvait déjà dans la théorie antérieure des principes et paramètres. Les principes constituent les caractéristiques définitoires du langage humain lui-même, de tout idiome existant ou possible. Ces caractéristiques sont organisées par un choix binaire entre deux paramètres. 
Le paramètre du sujet nul serait donc un des deux choix possibles du principe de l'instanciation du sujet (Larrivée sous évaluation). Toute langue doit instancier un sujet, mais ce sujet peut avoir une réalisation nulle, comme en italien (où on peut dire Ho parlato a Maria, soit littéralement "ai parlé à Marie»), ou une réalisation phonologique obligatoire, comme en français (où Ai parlé à Marie est normalement agrammatical face à J'ai parlé à Marie). Ces choix paramétriques seraient corrélés à la forme du paradigme verbal de la personne : toutes les personnes du verbe italien ont des marques distinctes, ce qui permettrait d'identifier le sujet et rendrait son expression redondante pour ainsi dire, expression nécessaire là où le sujet ne peut être récupéré, du fait d'un marquage partiel dans le verbe français par exemple de la personne. Cependant, la personne n'est marquée par aucun verbe en chinois, qui favorise pourtant l'absence du sujet. L'hypothèse d'un marquage distinct de chaque personne a été remplacée par celle d'un paradigme uniforme, qui manifeste ou bien toutes les oppositions, ou bien aucune d'entre elles. Généralisation en butte au contre-exemple de l'islandais ancien, entre autres, où le paradigme de la personne verbale n'est pas plus uniforme que dans la majorité des langues germaniques et où pourtant l'absence de sujet est acquise. Tant et si bien que l'absence du sujet apparaissait comme une propriété isolée. Cette propriété isolée n'est en outre pas toujours réalisée de façon catégorique. Ainsi, le sujet nul est permis dans certaines variétés de français et d'italien sous des conditions particulières, avec telle personne mais pas telle autre par exemple, comme l'a montré l'admirable travail de Heap (1999). Le caractère variable de ces réalisations est attesté à l'intérieur d'une même variété d'une langue : le français dit standard connaît des verbes (voilà, voici, Morin 1988, 1985 ) et des constructions (principalement impersonnelles, par exemple dans Il faut que soit mis fin à ces troubles ou Reste à voir si elle viendra) à sujet nul, ce qui en anglais se manifeste dans le style textuel particulier des recettes de cuisine (Roberge et Massam 1989), lors même que cette option est très généralement exclue dans ces langues. De plus, le sujet nul semble possible en français québécois avec certains auxiliaires à initiale consonantique, permettant peux pas et sont pas là mais non * ai pas et * est pas là (voir aussi le travail de Auger). Ces faits suggèrent que les options paramétriques constituent des choix non catégoriques, qui n'affectent pas l'entier d'une (variété de) langue, ce qui va à l'encontre de l'idée d'un ensemble uniforme de propriétés génériques.

Le caractère non catégorique des choix pourrait être contesté, bien entendu. On pourrait alléguer que les connaissances propres au langage interne (la compétence, la langue) demeurent uniformes et générales, que celui-ci n'est pas affecté par les vicissitudes du langage externe (la performance, la parole). Sans critères indépendants pour départager externe et interne et sans description précise du second, cette objection reste évidemment purement théorique. D'autres théories ont tenté de préciser les propriétés du langage interne de façon à prendre en compte les variations du langage externe. Ces variations dépendraient des rapports s'instaurant entre les différentes contraintes structurant la grammaire d'une communauté, selon la théorie de l'optimalité. Ces rapports supposent un ordre d'intervention ou une prégnance plus ou moins forte d'une règle par rapport à l'autre. Ces exigences potentiellement contradictoires permettent d'expliquer la possibilité de degrés de bonne formation, plutôt que les seules options d'un résultat soit bon, soit mauvais. La variation et le changement s'expliquent dans ce cadre par la modification des rapports entre les règles, ou les variations dans l'interprétation de leur application. Par exemple, selon Heap (dans une conférence présentée à l'AFLS en août 2000 à l'Université Laval), l'interprétation de l'instruction de placement des clitiques 
qui stipulerait que le terme le moins marqué apparait le plus à gauche expliquerait l'existence à la fois de le lui dire et de lui le dire en français, sans avoir à supposer deux systèmes syntaxiques différents: la première séquence reflète l'interprétation de la marque en termes de fonction (l'objet direct étant moins marqué que l'indirect), la seconde, celle en termes de sémantique (l'animé étant moins marqué que l'inanimé, voir me le dire versus *le me dire). Si l'interaction des règles donne des résultats séduisants, ce sont encore des règles catégoriques qui sont convoquées. Il faut se demander si l'application obligatoire d'une règle conçue comme générale n'est pas ce qui fait problème. Ce problème sera envisagé par la tension entre l'obligation et la simple possibilité qui a été envisagée dans un ensemble de disciplines contemporaines.

\section{L'obligé et le possible : analogies biologiques et culturelles}

15 La tension entre le hasard et la nécessité dans la constitution des rapports forme/sens trouve des analogies dans les représentations d'autres phénomènes symboliques considérés dans les sciences de l'humain. La sociologie et l'anthropologie culturelles ont reconnu assez tôt la discontinuité dans les représentations normatives ou idéologiques sous-tendant les pratiques d'un même groupe. S'éloignant de la proposition d'un projet unique qui caractériserait une culture chez Benedict et Mead, dans la notion de « personnalité de base » de Linton et de Kardiner (Cuche 2001 : 35-44), c'est la coexistence de différents projets selon les domaines d'action qui est aujourd'hui envisagée. Cette multiplicité des projets pour un même individu est évoquée par les Scollons dans leur important ouvrage sur l'analyse des relations interculturelles (2001). Ils y mentionnent que la caractérisation des individus appartenant à la culture chinoise comme étant plus polis que les Occidentaux ne correspond qu'à certaines situations discursives, et que les échanges commerciaux conventionnalisés sont conduits d'une façon qui pourrait sembler brutale. Ces différences marquées rappellent les manques apparents de cohérence des comportements: Cuche cite par exemple une étude sur des musulmans rigoristes des groupes Soninké et Toucouleur travaillant dans un abattoir de porc de Bretagne (2001: 63). Se retrouvant dans tous les groupes, ces incohérences apparentes sont analogues aux discontinuités qu'on retrouve dans les paradigmes linguistiques, et entre les paradigmes. L'idée de contingence est illustrée par celle de bricolage, défendue pour la constitution des ensembles de représentations culturelles par Lévi-Strauss dès 1963 (Cuche 2001 : 79) : les changements de représentations en cours ou achevés empruntent souvent des éléments sans rapport initial à l'ensemble où elles se trouvent intégrées. Ces réinterprétations sont à l'œuvre dans les processus de contact culturel, où de nouveaux éléments sont réintégrés à un ordre ancien, où les composantes d'un ordre ancien sont refondues en une signification, une fonctionnalité nouvelles.

Les sciences de la vie illustrent de plus la notion de contingence. La découverte en juillet 2002 au Tchad de Toumaï, fossile de 6 millions d'années, celle en 2001 à Dmanisi en Géorgie d'un autre hominidé vieux de 1750000 ans, remettent en jeu l'idée déjà contestée d'un développement linéaire de l'Homo sapiens, ou d'un foyer unique de son apparition. Cette contestation est déjà bien implantée en paléontologie, où l'abondance des fossiles différenciés pour les espèces les plus diverses laisse entrevoir une évolution foisonnante plus ou moins arbitraire plutôt qu'une progression stricte de développements séquentiels d'un niveau de complexité à l'autre. Le paléontologue Stephen Jay Gould est célèbre pour 
avoir mis en avant dans des ouvrages de vulgarisation l'idée d'une évolution fortuite sélectionnée par un environnement particulier. L'évolution peut donc venir d'un changement génétique spontané qui, une fois sanctionné par l'environnement immédiat, pourra être conçu comme une adaptation à cet environnement. L'environnement pourra également encourager ou décourager des changements de comportement qui auront un impact biologique et amèneront quelque adaptation. La notion d'adaptation ne peut pas expliquer les évolutions plus complexes cependant. Les modifications considérables que suppose l'apparition de l'aile par exemple ne peuvent provenir d'un changement génétique spontané unique, puisque le nombre de gènes à modifier est trop important pour que cette apparition soit plausible - et qu'en l'occurrence même de sa réalisation, elle empêcherait probablement la reproduction de l'individu devenu par voie de conséquence assez différent des membres des espèces les plus proches. Une apparition guidée par une série de changements génétiques successifs repose soit sur un hasard tout aussi improbable, soit sur la fonction finale de l'organe qui en justifierait le développement. Cette fonction n'étant cependant pas disponible pour les différents individus de l'espèce avant que l'organe ne soit développé complètement, l'émergence de ce dernier se trouve dépourvu de toute motivation pour les individus des stades initiaux du développement présumé : autrement dit, une aile ne peut être développée par un individu qu'en vue du vol; or, le vol n'est permis à un individu qu'à la fin du processus de développement de l'aile étendu sur plusieurs générations; donc, le développement de l'aile ne peut être motivé par le vol, même si c'est à cette fin que sera utilisée l'aile. Déjà soulevé contre Darwin, le problème des stades initiaux des modifications biologiques importantes amène Gould à proposer face à la notion d'adaptation celle d'exaptation. Il s'agit d'envisager l'utilisation d'organes complexes à une fin donnée comme le détournement de la finalité initiale qui en aurait motivé l'émergence. Ainsi, la finalité initiale des membranes ayant donné lieu aux ailes des insectes en aurait été une de conservation de la chaleur de l'individu. La fonction thermorégulatrice est optimalement satisfaite chez les petits insectes (de $2 \mathrm{~cm}$ ) par des membranes courtes qui ne donnent aucun avantage aérodynamique ; chez les insectes plus gros $($ de $10 \mathrm{~cm})$, le vol est rendu possible par des membranes assurant une chaleur optimale. Le simple changement de la taille de l'individu suffit à rendre utilisable à d'autres fins une propriété biologique développée avec un but original différent. La notion d'exaptation a deux conséquences pour le langage. D'une part, le langage humain pourrait être une exaptation de fonctions autres et rendue possible par la croissance de la taille du cortex d'hominidés devenus simplement plus grands. D'autre part, comme l'aile, le langage n'a pas toujours été une propriété génétique, puisqu'un ensemble coordonné de mutations spontanées ne peut expliquer le développement d'un phénomène aussi complexe, et donc que la pratique initiale du langage ne peut être motivée par le bagage génétique (arguments repris par Smith $2002: 90)^{3}$.

Ces détournements fonctionnels illustrent le bricolage qui préside à l'origine des individus et des espèces, que Gould baptise le principe du panda. Descendant de l'ours carnivore dont le pouce est atrophié, le panda actuel se sert d'un pouce fonctionnel pour la cueillette du bambou dont il se nourrit, pouce dont fait office un os sésamoïde du poignet hypertrophié. Le faux pouce du panda montre que les fonctions mêmes vitales peuvent être remplies par des caractères qui ne sont ni parfaits, ni parfaitement destinés à ces fonctions. Le caractère fortuit des espèces et de leurs propriétés implique que l'évolution ne suivrait pas nécessairement la même chronologie, ni n'arriverait aux mêmes résultats à partir de la même situation biologique initiale : l'existence de l'humain 
est ainsi un hasard parmi d'autres. Les choses sont comme elles sont parce qu'elles peuvent être ainsi, parce que rien de pressant ne s'y oppose, non parce qu'elles le doivent, comme le suggère Gould mais aussi Varela, Thompson et Rosch (1991 : 194ss) : on sait que c'est au hasard que s'établissent initialement les liens synaptiques, qui feront l'objet d'une sélection ultérieure, sélection réversible jusqu'à la septième année, voire plus tard. On sait aussi que l'arbitraire caractérise le rassemblement des gênes (dans celui qui associe les prédispositions à être gaucher et l'intolérance du colon au germe de blé, Varela, Thompson et Rosch 1991: 188), que les gênes sont loin de représenter un ensemble optimal (par l'existence de près de $40 \%$ de l'ADN qui ne porte aucune information, Varela, Thompson et Rosch (1991: 191), et par l'existence de gênes dits «transposons » qui répliquent la même information en plusieurs endroits du génome (Le Guyader 2002 : 93), et que leur sélection fait l'objet de compétitions au niveau moléculaire (comme avec les transposons), cellulaire (avec les cellules cancéreuses) et individuel (les mieux adaptés ou les plus habiles à se reproduire essaimant leur patrimoine génétique) (Le Guyader 2002 : 94). Ces bricolages biologiques attestent de la contingence des espèces et aident à penser celle des phénomènes intéressant les sciences du langage.

\section{Contingences et théorie de l'action : l'exemple du formulaire}

Comme le corps des informations biologiques ou des connaissances culturelles, les représentations linguistiques s'organisent de façon partiellement contingente. Les faits de contingence et de variation constituent une difficulté conceptuelle apparemment insurmontable pour les métaphores dominantes du système saussurien et du système computationnel chomskyen. Se retrouvant dans la plupart des cadres d'analyse actuels, ces métaphores supposent un ensemble de règles stables valables pour tous les membres d'une communauté. Cette perspective est ultimement motivée par la nécessité d'expliquer l'intercompréhension, et donc l'existence même du langage: si un idiome existe, c'est pour que ses locuteurs se comprennent, en conséquence de quoi ils possèdent les mêmes connaissances. Ces connaissances sont supposées systématiques, puisque l'absence d'organisation ne permettrait pas l'acquisition et l'usage rapide du langage. Les arguments d'acquisition et d'intercompréhension excluant et variation et contingence doivent être examinés. L'intercompréhension suppose-t-elle réellement des systèmes parfaitement semblables chez deux locuteurs? Après tout, des locuteurs de variétés d'une même langue arrivent tous les jours à se comprendre - un Québécois qui dira à un Français qu'il a arraché les tapis de son appartement sera compris parce que même si tapis en gallican ne permet pas de référer à de la moquette, arraché suppose une fixation qui permettra d'induire que c'est du référent de cette dernière qu'il s'agit et non du référent du premier. De plus, la compréhension n'empêche pas les expressions idiosyncrasiques - mon fils utilise depuis l'âge de trois ans depuis pour introduire des causales équivalentes à celles en puisque, sans que cet emploi existe dans son environnement et avant d'avoir su le premier mot d'anglais, tout cela sans causer d'incompréhensions dans les échanges. Les interactions ne supposent pas nécessairement une communion d'esprit ou de grammaire. Le terme de "communion" est employé sciemment, pour suggérer la vieille idée métaphysique d'une correspondance exacte entre chose et expérience, expérience et mot, mot et pensée. La croyance que le mot désigne exactement la chose alors qu'il n'en donne que des indications schématiques 
constitue un décalage important qu'avait déjà signalé Victor Henry au début du siècle dernier et qu'illustre l'exemple de billet. En lui-même, hors de tout contexte d'emploi particulier, le mot ne donne aucune référence particulière : on peut imaginer pour s'en convaincre l'exemple d'une feuille de papier trouvée dans un parc et qui ne contiendrait que ce mot. La référence à un objet devient immédiatement évidente dans le contexte d'un métro, d'un échange épistolaire ou d'une banque. Or, même si l'interprétant peut les associer immédiatement, il n'y a pas plus de correspondance métaphysique entre le syntagme "billet de train ", l'image d'un billet de train sur une publicité de la SNCF et l'objet concerné qu'entre la connaissance linguistique de deux individus. La même conclusion pourrait être atteinte avec d'autres lexèmes polysémiques, carte par exemple. Le rapport entre mot et chose est en outre médié par un environnement d'interaction; constitué de repères non linguistiques qui permettent de fixer l'interprétation, cet environnement fait en sorte qu'un ensemble parfaitement identique de connaissances linguistiques n'est pas nécessaire à l'intercompréhension. L'intercompréhension ne suppose pas non plus un ensemble systématique de règles suivant le même raisonnement : que l'expression des indéfinis soit proportionnelle ou non, que toutes les cases soient remplies et une seule fois, cela n'importe guère si le but est de communiquer. De même, le caractère systématique de l'input ne semble pas plus nécessaire à l'acquisition que le partage d'informations identiques. Ce partage est par définition impossible dans les termes initiaux et intermédiaires du processus d'acquisition (l'apprentissage du paradigme des indéfinis suppose un état où ils ne sont pas entièrement connus), laquelle peut se réaliser pour des ensembles plus ou moins uniformes (les différentes asymétries des ensembles linguistiques étant acquises sans difficultés apparentes).

L'existence à la fois de la contingence et de la variation et de l'intercompréhension demande une métaphore différente. Une telle métaphore peut être élaborée à partir de la notion d'action, qui peut aider à comprendre celle de communication. La communication ne se réduit pas aux traits linguistiques, elle s'appuie sur le gestuel et le facial, sur l'environnement d'échange, qui peut faire attendre un projet précis. Car c'est le projet qui structure les manifestations de la communication. On communique en vue d'un objectif particulier - sans projet, on ne communique vraisemblablement pas, c'est peut-être ce qui distingue crucialement le sujet parlant de l'ordinateur, et rend si difficile la production et l'acquisition automatique d'un discours non spécialisé, la machine n'ayant ni conscience, ni exigences matérielles, ni intérêts, ni instances de légitimation. C'est parce que ces différents besoins n'ont pas à être satisfaits dans les contextes formels par le médium de la langue étrangère que son acquisition donne de piètres résultats, comme en font foi la plupart des systèmes publics d'éducation occidentaux, et les résultats de l'acquisition de la langue maternelle par l'enfant sont spectaculaires en bonne partie parce qu'elle constitue un médium important pour la recherche de la satisfaction de ces besoins.

Une étude qui lie aux conditions d'usage la question de la contingence des faits linguistiques est fournie par Wray (2002) dans son ouvrage sur le formulaire. Le formulaire est le phénomène selon lequel une séquence est traitée comme un tout. Ce phénomène recouvre les chansons, les prières, les citations, les mnémotechnies et les listes, les proverbes et dictons, les routines interactionnelles (Eh ! ben, Bon Dieu !, Bonjour !, quinze heures quarante cinq plutôt que trois heures trois quarts), les figements (au fur et à mesure, pisser dans un violon), les constructions (Plus elle parle, plus je m'énerve et le Nous 
sommes plusieurs à le croire traité par Gaatone 2003), les collocations (en français, on prend une décision, qui n'est ni faite ni choisie) - collocations auxquelles on peut ajouter certaines conventionnalisations dans les rapports entre dérivés : un verbe comme prendre ne se nominalise en prise que dans une minorité de ses emplois, et prise d'une bière est bizarre face à prendre une bière; de même correctionnel ne s'applique qu'à la référence carcérale de corriger, non à celle concernant les copies par exemple; destination ne correspond qu'imparfaitement à destiner; Wray (2002: 265) cite Chomsky (1965:186) qui signale l'asymétrie entre horror, horrid, horrify ; terror, *terrid, terrify ; candor, candid, *candify. Ce type de phénomènes s'identifie par :

- (1) sa récurrence particulière dans l'emploi (bien que cela pose le problème de la délimitation de la séquence et de l'étendue souvent considérable du corpus nécessaire pour détecter ces récurrences),

- (2) l'effacement de distinctions internes (moins de pauses entre les items, réductions vocalique et consonantique), une certaine fixité (qui rend difficiles les reformulations comme la passivation, les déterminations par l'insertion d'un adjectif ou d'un adverbe - voir ?? J'ai pissé dans un violon rouge?? Le violon a été pissé dedans -, les variations morphologiques de nombre et de temps par exemple et les modifications de la structure intonative et accentuelle, fixité qui varierait avec la prégnance subsistante du sens littéral), des irrégularités (formes et structures isolées, au fur et à mesure, à qui mieux mieux),

- (3) l'absence de compositionalité (le sens du tout ne se déduisant plus du sens des parties critère qui pose la difficulté majeure d'inclure par définition les unités à valeur pragmatique, puisque la pragmatique est généralement conçue comme distincte du sens « littéral » associé au référentiel).

21 Ces comportements jamais catégoriques n'en reflètent pas moins le caractère unitaire des séquences formulaires. C'est parce qu'elles sont constituées en un signe par une relation unitaire entre une forme et un sens que leurs composantes peuvent présenter des idiosyncrasies. L'agrégation de ces composantes en un tout unitaire aurait un fondement interactionnel. L'interaction est présentée comme visant la promotion des intérêts du sujet. C'est au problème non linguistique de la promotion de soi qu'apporte une réponse linguistique le formulaire. Le formulaire présente l'avantage de réduire l'effort de production et d'interprétation d'un message par rapport à ce que demanderait le même message élaboré à partir d'unités librement assemblées. C'est le cas dans les situations d'interaction récurrentes comme l'ouverture et la fermeture de conversations. Les productions discursives plus exigeantes d'un point de vue psycholinguistique de même contiendraient plus de formules - dans la littérature orale (qu'on songe aux clichés homériques), dans les discours publics improvisés, dans les commentaires sportifs (ceux des sports plus rapides comme les courses hippiques contenant plus de formules que ceux de sports plus lents comme le cricket), dans les encans pour un public général. Ces formules donnent le temps au locuteur d'élaborer un message dont la production est temporellement contrainte, et lui permettent de soutenir les attentes stylistiques dont il est l'objet dans ces situations de communication.

22 En outre, en recourant à des formules pour lesquelles il existe des réponses comportementales prescrites, le locuteur limite les incompréhensions, mais aussi, l'éventail des réactions de l'interlocuteur et favorise donc le comportement désiré. L'obtention des comportements voulus par le moyen des routines interactionnelles fait en sorte qu'elles sont acquises très vite par les enfants: Wray donne l'exemple (2002: 107) d'une enfant de 23 mois qui pour demander un biscuit utilisait la séquence Time for a cup 
of coffee employée par sa mère au moment de prendre une pause pendant laquelle elle avait l'habitude de donner un biscuit à la fille. Les aptitudes linguistiques de cette dernière étant dépassées par cette séquence, elle ne pouvait avoir été apprise et produite que comme un tout. Une telle séquence à fonction interactionnelle n'a pas besoin d'être analysée, analyse qui n'apporterait aucun bénéfice, puisque n'en dépend pas l'atteinte des buts recherchés. L'analyse apparaît quand une séquence n'a pas en elle-même de fonction interactionnelle manifeste et que sa composition semble régulière. L'absence de régularités rend difficile et coûteuse l'identification des parties, même si elle est encouragée par l'apprentissage de l'écriture dans nos cultures. L'écriture promeut une approche analytique des inputs linguistiques chez les apprenants étrangers adultes. L'acquisition réussie de la langue cible dépend d'une interaction soutenue avec un environnement naturel d'échange, les contextes formels résultant en cette absence bien connue de sensibilité aux registres et à l'idiomaticité, cette sensibilité étant inutile puisque le langage ne sert pas à exprimer des besoins dans ce cas. À l'opposé, l'expression de ces besoins est rendue difficile dans les cas de dégénérescence linguistique, et les patients souffrant d'aphasie fluente (de Wernicke) leur imposant de sévères limitations de traitement tendent à ne recourir qu'à des constructions figées d'où manquent les expressions lexicales productives. Les seuls traits linguistiques qui subsistent dans les aphasies silencieuses (de Broca) sont, dans l'ordre, oui et non, les expressions émotives (exclamations et jurons), parfois les chansons et les prières, plus rarement les expressions d'interaction conversationnelles (les bonjour et les s'il vous plaît), laissant une incapacité générale de recourir à la stratégie analytique. Sur la base de ces observations, cinq localisations sont distinguées pour la mémorisation des unités conventionnelles par Wray, un lieu pour les unités grammaticales (morphèmes et mots outil), un second pour les unités référentielles (les unités et collocations), un troisième pour les figements et termes pragmatiques de routines interactionnelles, un quatrième pour les listes et un cinquième comprenant les expressions émotives, les deux premiers étant associés à l'hémisphère droit, les deux autres à l'hémisphère gauche et le dernier au sous-cortex. Le lexique serait ainsi distribué selon la fonction des termes qu'il contient, ce qui expliquerait que même si les expressions "créatives" ont le même statut d'unités conventionnelles que les expressions formulaires, ces dernières peuvent rester disponibles quand il y a lésion au cerveau droit contenant les premières. C'est ainsi qu'un aphasique peut utiliser la séquence fils de pute et se trouver incapable d'utiliser le nom fils pour désigner ses propres enfants (2002: 253). Cela signifie qu'un même signe peut se trouver mémorisé à différents endroits, et donc plus d'une fois, selon la fonction qu'il assume.

23 La biologie du langage n'est donc pas organisée de façon optimale, elle contient des redondances et des contingences qui se trouvent motivées par des considérations fonctionnelles. Le désir de voir ses besoins satisfaits, d'être écouté et pris au sérieux, de marquer son appartenance au groupe, d'induire des comportements sont des objectifs que permet d'atteindre le langage et qui se trouvent reflétés dans les unités qui le composent. Le formulaire en constitue un exemple, et le traitement holistique des unités comporte une efficacité et une économie que n'a pas le traitement créatif dans certains contextes. Ces deux traitements ont chacun leur pertinence, et l'approche holistique ne peut plus être considérée comme un phénomène marginal. 


\section{Théorie de l'action et contingences}

24 d'une pratique orientée vers des buts. L'action comme fondement de la représentation est un thème traité par un grand nombre de disciplines et de cadres d'analyse. En linguistique, le phonologique est envisagé comme un ensemble de représentations motrices liées à la production des sons par la motor speech theory (une proposition comparable a été faite pour le lexique, voir Allott 2002, qui rappelle l'idée plus générale des propriétés extrinsèques de Cadiot et Nemo), le lexique et la grammaire portent les traces de la situation d'interlocution (Benveniste) et des visées argumentatives (Ducrot), la pragmatique présente les inférences comme le résultat de l'interaction (Grice, Sperber), laquelle structure toute l'analyse de discours (voir Schegloff, Ochs et Thompson 1996) et la théorie des actes de langage. Dans les sciences humaines, depuis le sociologue Parson et jusqu'aux thérapies comportementalistes, l'action est donnée pour fondement de la représentation (voir Mutulesi 1998 par exemple). Biologiquement, représentation et action sont liées étant donné la constitution du système nerveux qui comme le suggère Varela conjoint motricité et fonctions cognitives (voir aussi Ito 2000). Un exemple intéressant à la croisée du biologique et du culturel est donné par le cas des émotions. Généralement considérées comme une réalité intime, les données émotives s'analysent en tant que le résultant de l'interaction qui prépare à l'action et cherche à faire agir les autres participants de l'interaction (Burkitt 2002). Autant un jugement négatif sur un état de fait que la tentative de détourner l'autre de quelque projet peuvent être servis par la colère. Ces émotions sont généralement conventionnalisées dans leurs manifestations extérieures, la conventionnalisation s'appliquant d'autant plus quand s'installe un décalage temporel entre une interaction et les réponses auxquelles elle donnera lieu. $\mathrm{Ce}$ sont ces réponses qui rendent possible l'émotion, si on veut en croire le témoignage de Damasio selon lequel le désespoir attendu n'est pas manifesté par les patients atteints d'un syndrome de paralysie totale (lock-in syndrome), qui, tout conscients qu'ils soient $\mathrm{du}$ tragique de leur situation, ne peuvent en marquer les manifestations (faciales, posturales, etc. Damasio 1999: 291ss); de même, l'impossibilité de percevoir (cécité), d'interpréter (autisme) ou à cause de différents troubles des muscles faciaux de produire les signes des émotions chez des sujets se corrèle à l'émoussement qu'ils rapportent de leurs vie émotive (Cole 2000). Ainsi, l'action peut être envisagée comme subsumant les différents modes de communication.

Le caractère expédient du langage semble la condition à la fois de la variation et de la contingence. L'argument est que puisque le langage n'est qu'un des mediums permettant d'atteindre un but qui seul intéresse l'individu, les variations et les irrégularités du medium sont sans importance tant qu'elles ne gênent pas l'atteinte du but. Le fait que des buts soient récurrents expliquent leur conventionnalisation. Rendue nécessaire par la faiblesse de l'instinct humain, cette institutionnalisation pourra suivre ou non des organisations systématiques, sans épuiser dans un cas comme dans l'autre le domaine des réponses qui peuvent être données aux problèmes de communication, c'est-à-dire d'accomplir chez et avec l'autre un projet. Le caractère conjoint de l'activité communicative explique que le langage sémiotise les conditions de son emploi, et toutes les marques potentielles des divisions sociales qui caractérisent ses usagers. L'inscription de la parole dans la langue est un fait connu, mais dont on n'a pas nécessairement tiré

Corela, 1-2 | 2003 
toutes les conséquences. La langue n'est pas qu'un répertoire de formes servant à construire des propositions, ce répertoire contient les conditions, les régulations de son usage en vue de l'atteinte d'un but (le mot sens en français peut aussi référer à la visée d'un objectif, et quelque chose a un sens quand il s'inscrit dans un projet: dire que la vie n'a pas de sens revient à exprimer qu'elle n'est pas dirigée par un projet transcendant). La visée de ce but ne semble pas la plupart du temps provenir d'une intentionnalité réfléchie, ce qui contredit la vision qu'a le sens commun de l'action, et pose la question de savoir quelle cause a l'action. Cette cause pourrait se trouver dans la notion de déclencheur des éthologues, qui voient maints comportements comme une réponse à des manifestations. Ces manifestations peuvent avoir une origine interne (les besoins physiologiques ou ceux symboliques que tente de traduire la théorie de faces par exemple) ou externe (environnement matériel, social). Ces origines opèrent rarement sans médiation culturelle, et cette culture suppose une socialité dont on peut attendre qu'elle marque les manifestations, mais non un ensemble parfaitement régulier de représentations se répondant également chez tous les membres de cette culture.

Cette perspective ne suppose pas autre chose que des idiolectes ajustables les uns aux autres dans l'interaction. La notion d'idiolecte est joliment documentée par Scollon (2002). Il montre qu'une expression nippone comme «chodai " "donne-moi » peut se retrouver dans les interaction qu'a une mère d'origine japonaise avec sa fille. Élevée en anglais, cette dernière n'en adoptera pas moins l'expression, comme le montrent les enregistrements par Suzanne Scollon. Celle-ci utilisera une fois l'expression deux ans plus tard avec sa propre fille, sans que ni l'une ni l'autre ne soit nipponophone. Cet exemple extraordinaire montre la circulation des formes (théorie des réseaux sociaux (Milroy), notions de communauté de pratiques (Meyerhoff), communauté de discours (Patrick), accommodation discursive de Giles), la fonction interactive des entités linguistiques, la contingence des moyens pour ces interactions, et le caractère idéologique de la notion de langue, puisque aucune des filles n'appartient à la langue ou à la nation japonaise. Les sources politiques de l'idéologie de l'unité d'une langue ne sont plus à discuter, mais reste à savoir ce qui en outre amène les citoyens et les linguistes à adhérer à cette croyance. L'intuition d'une communauté dans la pratique des locuteurs dérive sans doute de l'intercompréhension, qui est après tout le but de l'action communicative. Mais cette intercompréhension ne suppose pas une correspondance exacte entre les pratiques et les connaissances qui les soutiennent, ne nécessite pas une énigmatique grammaire commune, aussi énigmatique que l'inconscient collectif par exemple. La croyance en une langue strictement partagée peut aussi provenir des traces de l'interaction qui se conventionnalisent (marqueurs de groupes régionaux, historiques, professionnels) et la capacité à reconnaître une même norme prestigieuse (la pratique d'un des groupes). Bien sûr, les pratiques individuelles tendent à s'ajuster dans une communauté et ne présentent probablement jamais des divergences quantitativement ou qualitativement considérables, bien que des divergences puissent exister pour la bonne raison que la forme du marquage linguistique est seconde par rapport au projet poursuivi.

\section{La perfection est-elle possible?}

La langue, la compétence est ainsi une conventionnalisation de situations d'interactions, de stratégies pour construire des discours, c'est un expédient en vue d'actions qui le dépassent. Ces actions marquent le corps des connaissances linguistiques de tout un 
ensemble de phénomènes idiosyncrasiques. Ces idiosyncrasies sont rendues possibles par le caractère instrumental et expédient du langage, car on ne gagne rien à tenter de donner à un outil qui suffit à une tâche une organisation parfaitement symétrique, et on peut même se demander si la symétrie est possible pour un simple moyen. La notion de perfection est elle-même problématique, et est envisagée dans le programme minimaliste d'un point de vue théorique interne dans l'applicabilité virtuellement générique des processus syntaxiques. Leur mise en œuvre concrète est cependant limitée à la fois dans l'extension des cas qu'ils concernent et par les emplois formulaires. Un corps de connaissance ne semble d'ailleurs pas pouvoir être parfait. La perfection ne se retrouve pas plus dans les langues artificielles, et le paradigme négatif de l'espéranto donne une forme indirecte pour personne mais non apparemment pour rien, donne une forme pour la quantité mais non pour la qualité (que concernerait Je n'ai rien fait de la journée, soit 'rien de spécial') :

(4) ne 'non', neniu 'personne', nenio 'rien', nenies 'à / de personne', nenia 'd'aucune sorte', neniel 'en aucune manière', neniam 'jamais', nenie 'nulle part', nenial 'sans raison', neniom 'aucune quantité'.

(http://perso.club-internet.fr/vivilass/cours/grammaire.htm)

Le paradigme des relatifs par exemple ne pourrait de même être symétrique. Même en admettant une relation proportionnelle entre la fonction et la catégorie sémantique d'(in)animé (supposons qu'il y aurait un qui1 pour le sujet animé, un qui2 pour le sujet inanimé, un que1 pour le complément direct animé et un que2 pour le direct inanimé), cette symétrie ne peut être conservée bien longtemps : qu'en serait-il du complément indirect, par exemple? Faudrait-il que ce complément soit rendu par un seul terme? Comment savoir alors si ce terme - appelons-le qua - doit dans La fille qua Paul a parlé être interprété comme la fille de Paul, la fille à qui Paul a parlé, de qui il a parlé ou pour qui il a parlé ? Le fait d'intégrer la préposition ne lève pas toutes les ambiguïtés, et le relatif dans $L a$ fille de qua Paul a parlé peut concerner le sujet ou le verbe. De plus, ces spécifications ne sont pas particulièrement économiques ou symétriques au vu des compléments directs. Qui plus est, les choix en éliminent d'autres: en anglais, les possessifs clitique et déterminant s'excluent mutuellement en coordination, et exprimer l'idée de la conférence de Paul et Mary doit donner lieu à une construction d'évitement: The conference organised by Paul and Mary plutôt que par ?? His and Mary's conference; de même, la négation d'un grand nombre d'occurrences ou d'un grand nombre d'occurrences diverses voient leur expression limitée par les (in)compatibilités entre aucun, plusieurs et différentes: on dira pas une seule de ces nombreuses conférences et non? aucune de(s) plusieurs conférences, plusieurs conférences différentes plutôt que *plusieurs diverses conférences. Et ce n'est pas tout, puisque à côté des distinctions morphosyntaxiques se trouvent celles de la sémantique. La catégorie des (in)animés ne résout pas d'elle-même en français le problème de savoir, par exemple, dans quelle classe mettre des agents non humains comme le chien ou la marée. Ces problèmes se multiplient quand il s'agit de combiner le sens à la forme. C'est parce que plusieurs niveaux de représentation sont supposés par le langage (sociaux, interprétatifs, syntaxiques, morphologiques etc, comme le suggère aussi Givón 1999) qu'un système parfait n'est probablement pas praticable : il n'y a pas moyen de produire un paradigme parfait des relatifs, puisque chaque choix à chaque niveau en exclut un autre, et qu'il semble impossible de relier ces choix de façon systématique à tous les autres plans. Il y a multiplicité des choix à chaque niveau, et multiplicité des niveaux de représentation du langage, et cette multiplicité s'explique par le besoin, la capacité, l'utilité de sortir la référence des cadres de l'interlocution" 
Une théorie de la grammaire et du langage comme mode d'action ne suppose pas nécessairement que toute règle est exclue, tout au contraire. Il n'est pas douteux qu'il existe des règles par défaut relativement générales, en morphologie (le fameux exemple de la conjugaison le montre, la production régulière du passé anglais en -ed surgénéralisée sur des verbes dont le passé a une forme idiosyncrasique comme le went de go, qui devient chez les enfants goed, abondamment discuté par Pinker ; sontaient et tiendre donnent des exemples analogues), en syntaxe (le français moderne ne permet normalement pas l'absence de sujet, et jamais l'absence de sujet référentiel), en sémantique (les déverbaux sont généralement interprétables comme action ou résultat de l'action, l'imparfait est normalement compris comme un imperfectif, quelqu'un comme un existentiel). De même, des tendances comme celle en français de ne pas incorporer la manière aux lexèmes verbaux, comme l'observait Emonds, pourraient expliquer le statut incertain des indéfinis de manière. Enfin, l'existence de formes conventionnelles rend celle de séquences créatives plus marquées. Ces généralités sont une des deux approches possibles qui dans certains contextes peuvent diminuer l'effort d'acquisition et de traitement. Simplement, l'approche analytique ne stipule pas des généralités nécessaires, mais bien des rapports possibles. C'est probablement l'existence de processus apparemment opposés dans une même langue qui servent de point de départ au changement, en donnant un modèle qui pourra être imité, et c'est peut-être à cela que pensait Troubetzkoi en disant que toute langue contient « des tendances qui développées, déséquilibrent le système linguistique et permettent sa reconstitution selon une structure nouvelle » (cité par Chiss et Puech 1997 : 202).

En même temps, la nécessité s'applique à certaines relations. Le paramètre de linéarisation qui rend possible la dissociation entre le caractère structurellement dominant du sujet et le caractère solidaire de l'argument et du prédicat, le paramètre de structuralité semblent des contraintes universelles du langage humain. Alors qu'un système connexionniste apprendra sans difficulté un marquage reposant sur le nombre de mots dans une séquence (un morphème sur chaque troisième mot de toute phrase par exemple), ce genre de marquage ne se retrouve jamais dans les langues naturelles et n'est acquis qu'imparfaitement et avec la plus grande difficulté par des sujets (Smith 2002). Authier et Reed (1999) cite encore le fait que l'interrogation fait monter le verbe dominant de la phrase, non le premier de la séquence, et on a donc Would people who are not concerned avoid intervening? et jamais * Are people who not concerned would avoid intervening?.

\section{Conséquences heuristiques d'une théorie de l'action}

31 Si des contraintes générales pèsent sur la forme possible des grammaires, ces grammaires ont une latitude considérable dans l'agencement des relations formes / sens des unités qui les constituent. Cette latitude s'explique par la multiplicité des niveaux de représentation, les informations de chacun de ces niveaux ne pouvant être associées sur des bases parfaitement symétriques. Un système de relations parfaites serait de plus superflu, puisque ces informations ont une finalité interactive en vue de l'atteinte de buts sociaux. Le caractère expédient du langage signifie qu'il s'accommode fort bien d'irrégularités diverses, qui pourront donc différer d'un individu à l'autre, ce qui ne gênera cependant pas leur aptitude à se comprendre, en particulier dans des contextes d'interaction balisés. 
modèle instrumental du langage se révèle supérieur à ceux du système saussurien et $\mathrm{du}$ système computationnel minimaliste en rendant possible l'explication de la contingence de la variation, du changement. Cette explication repose sur l'idée simple que sont actualisées des possibilités plutôt que des obligations. À cet égard, la notion d'autorégulation, par exemple, signifie tout simplement qu'un système complexe satisfait d'une façon ou de l'autre les fonctions qui lui sont nécessaires; si ces fonctions ne sont plus satisfaites, il n'y a tout simplement plus système. L'identification de ces fonctions nécessaires semble donc plus aisée que celle de la diversité de leurs réalisations. Ces perspectives emportent des conséquences heuristiques importantes pour la recherche linguistique. D'une part, la recherche de déterminismes généraux doit rester l'objectif scientifique premier. D'autre part, la découverte de règles non catégoriques, de proportions partielles et de stipulations ne devrait pas étonner si les observations présentées ici sont bien interprétées. Certains faits sont relativement généraux (la règle par défaut d'expression du sujet en français), certains faits le sont moins (la possibilité de la non-réalisation du sujet impersonnel dans certaines subordonnées), tous deux méritent au même titre l'intérêt de la recherche, qui n'a nul droit d'ignorer l'un ou l'autre. Une attention portée à un seul de ces ordres de faits pourra avoir des conséquences théoriques et pratiques fortement indésirables : toute la langue n'est pas stipulation, mais elle n'est pas non plus généralisation. Après tout, personne ne songe à poser la question de savoir pourquoi la consonne /p/ est sourde en français; elle est sourde de façon purement contingente. Personne ne songe non plus à donner une stipulation pour chaque manifestation du phénomène de dénasalisation des voyelles finales des adjectifs féminins, car le passage de un bon récit à une bonne histoire n'est pas lié à l'adjectif bon en particulier. L'équilibre entre généralisation et stipulation est évidemment une question que doivent en dernière instance trancher les faits, mais l'étude de ces faits ne peut plus ignorer les deux modes sous lesquels peuvent s'inscrire les connaissances linguistiques.

\section{BIBLIOGRAPHIE}

ALLOTT, R. (17.08.2002). Words for the apple 2. Linguislist 13, 2121.

AUGER, J. (1995). Pronomial Clitics in Quebec Colloquial French: A Morphological Analysis. Thèse de doctorat, University of Pennsylvania.

AUTHIER, J.-M. et REED, L. (1999). Structure and Interpretation in Natural Language. Munich : Lincom Europa.

BAKALLA, M. H. (2002). «What Is a Secret Language ». D. B. PARKINSON, D., B. et BENMAMOUN, E. (dirs). Perspectives on Arabic Linguistics XIII-XIV: Papers from the Thirteenth and Fourteenth Annual Symposia on Arabic Linguistics. Amsterdam et Philadelphie : Benjamins. 171-183.

BLANCHE-BENVENISTE, C. (2002). «Quelqu'un, quelque chose, quelque part, quelquefois ». Présentation à la conférence internationale Grammaticalisation en français, ENS, 17-19 janvier 2002. Document d'accompagnement, 6 pages. Article à paraître dans COMBETTES, B., MARCHELLONIZIA, C. et PREVOST, S. (dirs). Verbum. 
BURKIT, I. (2002). « Complex Emotions: Relations, Feelings And Images In Emotional Experience ». BARBALET, J. (dir.). Emotions and Sociology. Oxford: Blackwell. 151-168.

CHISS, J.-L. et PUECH, C. (1997). Fondations de la linguistique. Bruxelles : Duculot.

CHOMSKY, N. (1965). Aspects Of The Theory Of Syntax. Cambridge, Mass. : MIT Press.

COLE, J. (2000). « Living With Difficulties Of Facial Processing. Some Ontological Consequences Of Clinical Facial Problems ». Pragmatics and Cognition 8,1. 237-260.

CUCHE, D. (2001). La Notion de culture dans les sciences sociales. Paris : Découverte.

DAMASIO, A. R. (1999). The Feeling of What Happens. Body and Emotion in the Making of Consciousness. New York : Harcourt Brace.

DAMOURETTE, J. et PICHON, E. (1940). Des Mots à la pensée. Essai de grammaire de la langue française. Paris : d'Artrey.

DE MAURO, T. (1969). Une Introduction à la sémantique. Paris : Payot.

GAATONE, D. (2003). « Nous sommes plusieurs à participer à ces mélanges ». MS, 15 pages.

GIVON, T. (2002). «Bio-Linguistics ». The Santa Barbara lectures. Amsterdam et Philadelphie : Benjamins.

GIVON, T. (1999). « Generativity and Variation: The Notion 'Rule of Grammar' Revisited ». MacWHINNEY, B. (dir.). Emergence of Language. Hillsdale : Earlbaum.

GOULD, S., J. (1991). Not Necessarily a Wing. Bully for Brontosaurus. Further Reflections in Natural History. Londres : Penguin. 145-151.

GRUSH, R. (2001). « The Architecture of Representation ». BECHTEL, W., MANDIK, P., MUNDALE, J. et STUDDLEBEAM, R. (dirs). Philosophy and the Neurosciences. Oxford : Blackwell.

HEAP, D. (1999). La Variation grammaticale en géolinguistique : les pronoms sujet en roman central. Munich : LINCOM Europa.

ITO, M. (2000). « Neural Control of Cognition And Language ». MARANTZ, A., MIYASHITA, Y. et O'NEIL, W. (dirs). Image, Language, Brain: Papers from the First Mind Articulation Project. Cambridge : MIT Press.

KNIGHT, C. et al (dirs). (2000). Evolutionary Emergence of Language. Cambridge : Cambridge University Press.

LARRIVEE, P. Sous évaluation. «Contribution à un bilan méthodologique de la syntaxe de l'émergence diachronique du sujet obligatoire en français : le paramètre du sujet nul et le statut des clitiques ». MS, Aston University. 15 pages.

LASS, R. (1997). Historical Linguistics and Language Change. Cambridge : Cambridge University Press. LE GUYADER, H. (2002). « La théorie synthétique de l'évolution revisitée ». Pour la science 300. 92-95.

MONOD, J. (1970). Le Hasard et la nécessité. Paris : Seuil.

MORIN, Y.-C. (1988). « French voici And voilà: A Reply To Bouchard ». Language 64,101-103.

MORIN, Y.-C. (1985). « On The Two French Subjectless Verbs voici And voilà ». Language 61, 777-820.

MULLER, C. (1991). La Négation en français. Genève : Droz. 
MUTELESI, E., M. (1998). Subjectivité comme auto-organisation. Une étude du constructivisme radical au départ de Husserl. Thèse de doctorat, Université Catholique de Louvain. http://www.univie.ac.at/ constructivism/books/mutelesi/3.htlm

NYCKEES, V. (2003). « La perspective médiationniste en linguistique ». SIKSOU, M. (dir.). Variation, construction et instrumentation du sens. Paris : Hermès.

RIEDER, A. (2002). Beiläufiger Vokabelerwerb : Theoretische Modelle und empirische Untersuchungen. Thèse de doctorat, Université de Tubingue.

ROBERGE, Y. et MASSAM, D. (1989). « On Recipe Context Null Objects in English ». Linguistic Inquiry 20, 134-139.

SCHEGLOFF, E. A., OCHS, E. et THOMPSON, S., A.. (1996). « Introduction ». Interaction and Grammar. Cambridge : Cambridge University Press.

SCOLLON, R. (2002). « Cross-Cultural Learning And Other Catastrophes ». KRAMSCH, C. (dir.). Language Acquisition and Language Socialization. Ecological Perspectives. Londres et New York: Continuum. 175-196.

SCOLLON, R. (2001). Mediated Discourses, The Nexus of Practice. London : Routledge.

SCOLLON, R et SCOLLON, S., W. (2001). Intercultural Communication: A Discourse Approach. Oxford : Blackwell.

SMITH, N. (2002). Language, Bananas \& Bonobos. Linguistic Problems, Puzzles and Polemics. Oxford : Blackwell.

TURKELL, W. (2002). « The Learning Guided Evolution Of Natural Language ». BRISCOE, T. (dir.). The Linguistic evolution through language acquisition: Formal and computational models. Cambridge : Cambridge University Press.

VAN LIER, L. (2002). « An ecological-semiotic perspective », KRAMSCH, C. (dir.) Language Acquisition and Language Socialization. Ecological Perspectives. Londres et New York : Continuum.

VARELA, F. J., THOMPSON, E. et ROSCH, E. (1993). The Embodied Mind. Cognitive Science and Human Experience. Cambridge : MIT Press.

WRAY, A. (2002). Formulaic Language and the Lexicon. Cambridge : Cambridge University Press.

\section{NOTES}

1. Cet article a bénéficié des observations de ma collègue Dominique Lagorgette et de deux évaluateurs anonymes. Qu'ils trouvent ici l'expression de mes remerciements.

2. Je ne suppose pas que ces conséquences sont nécessairement reflétées dans le travail de Saussure lui-même; comme le rappelle un des deux évaluateurs anonymes de cet article, Saussure «limite la régularisation à ce que peuvent produire les principes généraux de la sémiologie : ça n'exclut certainement pas des paradigmes imparfaits. Il y a tout un discours sur la «valeur » où Saussure suppose nommément que des objets linguistiques irréguliers puissent jouer le rôle des formes régulières." Néanmoins, le travail d'une part importante des structuralistes va plus ou moins loin dans le sens de ces conséquences.

3. Une inscription génétique de systèmes linguistiques d'abord élémentaires se complexifiant progressivement vers des versions plus élaborées éventuellement inscrites est une hypothèse discutée par Turkel (2002), laquelle ne résout cependant pas la difficulté de la pratique nécessairement antérieure à l'organe de langage qui devrait la rendre possible. 
4. Van Lier (2002) semble suggérer, à partir des grandes distinctions de la sémiotique, l'hypothèse que la grammaire émerge avec la prédication (symbolique), à la suite de l'indexicalité et de la phase initiale de la réciprocité (iconique).

\section{RÉSUMÉS}

Cet article présente une réflexion sur le rôle des faits contingents pour la compréhension générale du langage. Ces faits sont illustrés par l'absence de proportionnalité dans le paradigme des indéfinis du français et par l'absence de corrélations stables entre le Paramètre du Sujet Nul et les autres paramètres. Ces irrégularités vont à l'encontre de ce que laissent attendre certaines versions du structuralisme et le Programme Minimaliste chomskyen actuel. Des perspectives développées dans les sciences de la vie et de la culture et dans des travaux récents sur le langage formulaire permettent d'envisager la contingence comme résultant du caractère expédient d'un médium tourné vers l'action. Une compréhension du langage en termes d'une théorie de l'action permet de retrouver le lieu de passage entre les règles par défaut qui structurent une grammaire de façon générale et les stipulations qui les actualisent dans l'usage particulier, ces deux modes jouant un rôle complémentaire dans l'organisation de l'information linguistique.

This paper is a reflection on the role of contingent facts for the general understanding of language. Such facts are illustrated by the lacks of proportionality in the paradigm of French indefinites and the irregular correlations between the Null Subject Parameter and other hypothesised parameters. Such contingencies clearly go against the expectation raised by at least some versions of structuralism and the current chomskyan Minimalist Program. As demonstrated by alternative views being developed in various natural and social sciences, and as shown by recent research on formulaic language, contingency may be understood as the result of the expedient character of a medium geared towards action. A view of language as action may thus offer a perspective able to account both for the general default rules shaping a grammar and for the contingencies that entrench them in use, as both are integral and complementary aspects of language.

\section{INDEX}

Mots-clés : contingences, théorie de l'action, indéfini, structuralisme, paramètre du sujet nul, programme minimaliste

\section{AUTEUR}

\section{PIERRE LARRIVÉE}

Aston University (United Kingdom) 